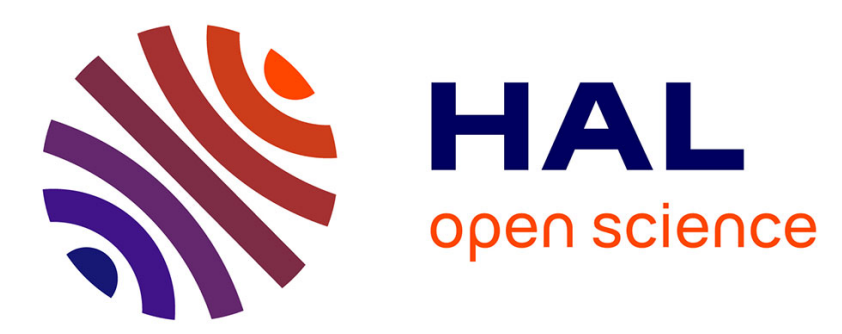

\title{
Multiscale Estimation of the Fluctuations of Mechanical Fields in Poroelasticity: Application to Cement-Based Composites
}

Tulio Honorio, Laurent Brochard

\section{- To cite this version:}

Tulio Honorio, Laurent Brochard. Multiscale Estimation of the Fluctuations of Mechanical Fields in Poroelasticity: Application to Cement-Based Composites. Sixth Biot Conference on Poromechanics, Jul 2017, Paris, France. 10.1061/9780784480779.164 hal-01686268

\section{HAL Id: hal-01686268 https://hal.science/hal-01686268}

Submitted on 20 Jun 2018

HAL is a multi-disciplinary open access archive for the deposit and dissemination of scientific research documents, whether they are published or not. The documents may come from teaching and research institutions in France or abroad, or from public or private research centers.
L'archive ouverte pluridisciplinaire HAL, est destinée au dépôt et à la diffusion de documents scientifiques de niveau recherche, publiés ou non, émanant des établissements d'enseignement et de recherche français ou étrangers, des laboratoires publics ou privés. 


\title{
Multiscale estimation of the fluctuations of mechanical fields in poroelasticity: application to cement-based composites
}

\author{
Tulio Honorio, Ph.D. ${ }^{1}$ and Laurent Brochard, Ph.D ${ }^{2}$ \\ ${ }^{1}$ Laboratoire Navier, UMR 8205, Ecole des Ponts, IFSTTAR, CNRS, UPE, Champs-sur-Marne, \\ France; e-mail: tulio.honorio-de-faria@enpc.fr \\ ${ }^{2}$ Laboratoire Navier, UMR 8205, Ecole des Ponts, IFSTTAR, CNRS, UPE, Champs-sur-Marne, \\ France; e-mail: laurent.brochard@enpc.fr
}

\begin{abstract}
Analytical estimations of stresses and strains fluctuations (or statistical variability) in elasticity at early-age are provided according to different scales of interest of cement-based materials (CBM). The estimations are obtained by means of relations between square means and effective properties for isotropic medium. Mori-Tanaka (MT), Self-Consistent (SC) and Generalized SelfConsistent (GSC) schemes are combined to represent the multiscale microstructure of CBM. In the estimations, the evolution of volume fractions of phases due to cement hydration processes is accounted for at the cement paste level. Interfacial Transition Zone (ITZ) is considered at mortar level. These estimations provide important information regarding the heterogeneity of the mechanical fields in cement-based materials, which can be used, for instance, in the definitions of failure or onset of damage criteria. The distributions of stress and strains are provided for the phases of interest at a given scale for different loadings.
\end{abstract}

\section{INTRODUCTION}

Cement-based materials (CBM) have a heterogeneous and hierarchical character, which is intimately related to key properties (elastic moduli, strength, conductivities) affecting its thermomechanical performance. Moreover, in these materials, we find a multiscale porosity with pore sizes ranging from few Angstroms (in the interlayer space between C-S-H sheets) up to few millimeters (in air trapped voids). Homogenization techniques, notably in poroelastic context, have been employed both to upscale macroscopic properties of interest in industrial application and to estimate by inverse analysis (or relaxation) the properties of some basic constituent phases of CBM. In general, these studies are limited to first-order estimations, i.e. only the averages of mechanical fields in a given phase are taken into account.

Local information regarding the deviation of these fields might be, though, useful information for the development of more precise estimations (especially in the case of strength 
and damage evolutions - in which a stress or strain criterion is often required). Indeed, fluctuations in a composite play a major role in the determination of failure mechanisms (Ponson 2016). For CBM, this local information have been obtained in some studies by means of numerical tools (e.g. with finite elements analysis (Honorio et al. 2016)). However, classical homogenization techniques can also provide analytical estimations of the fluctuations of strain or stresses fields in composites (Kreher and Pompe 1985). These analytical estimations can be more easily transposable to the study of different aspects related to CBM as, for instance, the composition (e.g. w/c, volume of aggregates) and hypothesis regarding the representation of the microstructures (the presence of an interfacial transition zone (ITZ), polycristal-like structure compared to matrix-inclusion structure).

We start from a given representation of the multiscale character of CBM combining matrix-inclusions morphologies according to classical homogenization schemes (Honorio et al. under review). Then, we use estimations of the variance of strain and stresses fields for these schemes (Bobeth and Diener 1987). We assume micro and macro isotropy. The estimations of fluctuations are made for spherical (or equiaxed) inclusions. As results, we present fluctuations within the phases defined in cement paste, mortar (including ITZ) and concrete scales according to different types of loading.

\section{OVERALL AND LOCAL BEHAVIOR IN THE PRESENCE OF TRANSFORMATION FIELDS}

Consider a composite with elastic constituent phases, which are perfectly bonded at their interfaces. We assume that the properties of the phases are statistically independent quantities. Assuming that such composite is homogeneous at a given macro-scale (so that a representative elementary volume (RVE) $\Omega$ can be chosen to study local and overall behavior), the overall behavior is, in terms of Cauchy stress $\sigma$ and linearized strain $\varepsilon$, respectively (Dvorak and Benveniste 1992):

$$
\sigma(\mathbf{x})=\mathbb{C}(\mathbf{x}): \varepsilon(\mathbf{x})+\sigma^{(0)}(\mathbf{x}), \quad \forall \mathbf{x} \in \Omega
$$

where $\mathbb{C}$ is the elastic stiffness symmetric fourth-rank tensor and $\sigma^{(0)}$ is the overall stress transformation field (or eigenstress). This affine form with the presence of transformation fields can be used to model different physical phenomena including thermo- and poro-elasticity as well as phase transformation. In saturated poroelasticity the transformation stresses read:

$$
\sigma^{(0)}=-\mathbf{B}(\mathbf{x}) P
$$

where $P$ is the pressure in the fluid filling the pores and $\mathbf{B}$ is the tensor of Biot coefficients.

Consider now a sub-volume $\Omega_{s}$ of the RVE. The local constitutive equation reads:

$$
\sigma(\mathbf{x})=\mathbb{B}(\mathbf{x}): \Sigma+\sigma^{r e s}(\mathbf{x}), \quad \forall \mathbf{x} \in \Omega_{s}
$$

where $\mathbb{B}$ is the stress localization forth-rank tensor; $\Sigma$ is a uniform stress applied at the boundary $\partial \Omega$ of the RVE and $\sigma^{r e s}$ is the residual stress field resulting from the incompatibility of the local eigenstrains. Adopting a mean-field approach one might write the localization expression for a given phase $p$ : 


$$
\langle\sigma\rangle_{p}=\langle\mathbb{B}\rangle_{p}: \Sigma+\left\langle\sigma^{r e s}\right\rangle_{p}
$$

With this equation the interphase distribution of the stress within a composite is obtained. In order to estimate both volume averages in the second term of last equation, we can resort to the well-known Eshelby solution for an ellipsoidal inclusion in an infinite homogeneous linear medium under a homogeneous loading $\Sigma$. Finally, it can be shown (Bornert et al. 2001; Laws 1973) that the average residual stress tensor can be obtained from the effective properties of the medium and the averaged stress localization tensors.

\section{FLUCTUATIONS OF MECHANICAL FIELDS}

Besides averaged information per phase or interphase distribution of fields, more precise local information regarding the intraphase estimations of the distribution of fields can be obtained by mean of analytical methods. Let the fluctuation or covariance (forth-rank) tensor of a field $\phi$ within phase $p$ be defined by

$$
q_{p}^{\phi} \equiv\langle\phi \otimes \phi\rangle_{p}-\langle\phi\rangle_{p} \otimes\langle\phi\rangle_{p}
$$

where $\otimes$ denotes the dyadic product. Note that, even if the constituents are isotropic, the fluctuation field is anisotropic per se (Bobeth and Diener 1986), for instance, under uniaxial loading, $q_{p}^{\phi}$ is significantly stronger in the direction of loading. The standard deviation $\omega_{p, i j k l}^{\phi}$ of a field $\phi$ within a phase $p$, according a considered direction, can be approximated by the square root of the components of the fluctuation tensor (Bobeth and Diener 1987): $\omega_{p, i j k l}^{\phi}=$ $\sqrt{q_{p, i j k l}^{\phi}}$. In Eq. (1), the rightmost term, considering the case of stress fields, is the average stresses $\langle\sigma\rangle_{p}$ per phase that can be obtained as shown in the last section. The term $\langle\sigma \otimes \sigma\rangle_{p}$, can be obtained by the definitions of local and effective elastic energies, $u$ and $U$, respectively (Brenner et al. 2001):

$$
\begin{gathered}
u(\mathbf{x})=\frac{1}{2}\left[\sigma(\mathbf{x}):\left(\varepsilon(\mathbf{x})-\varepsilon^{(0)}(\mathbf{x})\right)\right]=\frac{1}{2}[\sigma(\mathbf{x}) \otimes \sigma(\mathbf{x})]:: \mathbb{S}(\mathbf{x}) \\
U(\mathbf{x})=\langle u(\mathbf{x})\rangle=\frac{1}{2}\left[\Sigma: \mathbb{S}^{e f f}: \Sigma-\left\langle\sigma^{r e s}(\mathbf{x}): \varepsilon^{(0)}\right\rangle\right]
\end{gathered}
$$

where the effective elastic energy is obtained from the local energy by applying Hill's lemma. The second moment of stress field can be then obtained by deriving $U$ with respect to the local compliance $\mathbb{S}_{p}$ (Brenner et al. 2004; Buryachenko and Rammerstorfer 1998):

$$
\langle\sigma \otimes \sigma\rangle_{p}=-\frac{2}{f_{p}} \frac{\partial U}{\partial \mathbb{S}_{p}}=\frac{1}{f_{p}}\left[\Sigma \otimes \Sigma:: \frac{\partial \mathbb{S}^{e f f}}{\partial \mathbb{S}_{p}}+\left\langle\varepsilon^{(0)}\left(2 \Sigma: \frac{\partial^{t}\langle\mathbb{B}\rangle_{p}}{\partial \mathbb{S}_{p}}+\frac{\partial\left\langle\sigma^{r e s}\right\rangle_{p}}{\partial \mathbb{S}_{p}}\right)\right\rangle\right]
$$

The second moment of strain fields can also be obtained considering a uniform strain field on $\partial \Omega$ (Brenner et al. 2001). So far, these estimations of the second moments are valid irrespectively of any degree of anisotropy. In the micro/macro-isotropic case, for spherical inclusions, the expressions are reduced to the ones presented by (Bobeth and Diener 1986) in the absence of transformation fields or (Bobeth and Diener 1987) in the presence of transformation/residual 
fields. The latter expressions are used hereafter to estimate the fluctuations of mechanical fields in CBM.

\section{APPLICATION TO CBM}

Estimations of fluctuation in undrained Calcium Silicate Hydrates (C-S-H). We first consider the simple example of a porous material with saturated pores. A polycristal-like microstructure with equiaxed grain is assumed so that Eshelby solution can be employed. We employ this simple morphology to represent C-S-H as a function of different packing densities as shown in Fig. 1. For a hydrostatic loading, the maximum fluctuations in the solid arise at a packing density of ca. 0.55 and in the pore at 0.5. Following (Constantinides and Ulm 2004), the packing densities of 0.64 and 0.76 corresponds to Low Density (LD) and High Density (HD) C$\mathrm{S}-\mathrm{H}$, respectively. Undrained conditions and non-connected pores are assumed so that the fluctuations within pores can be assessed even in the absence of transformation fields (as a consequence, for a bulk modulus of water equal to $2.18 \mathrm{GPa}$, the percolation threshold of selfconsistent scheme is close to 0.4 ). Note that in the absence of macroscopic load, fluctuations may arise from the residual stresses due to transformation fields. In a homogeneously distributed packing density, LD solid C-S-H and corresponding pores are subject to larger fluctuations compared to HD C-S-H. Note that the fluctuations in solids within two standard deviations are large enough to involve opposite sign loading (traction if $\sigma_{0}$ is compression and vice-versa) for low packing densities $(<0.64)$.

The fluctuations are loading dependent. Figure 2 shows the fluctuations in solids for LD C-S-H according to 4 different loadings. Loadings involving shear present larger fluctuations.

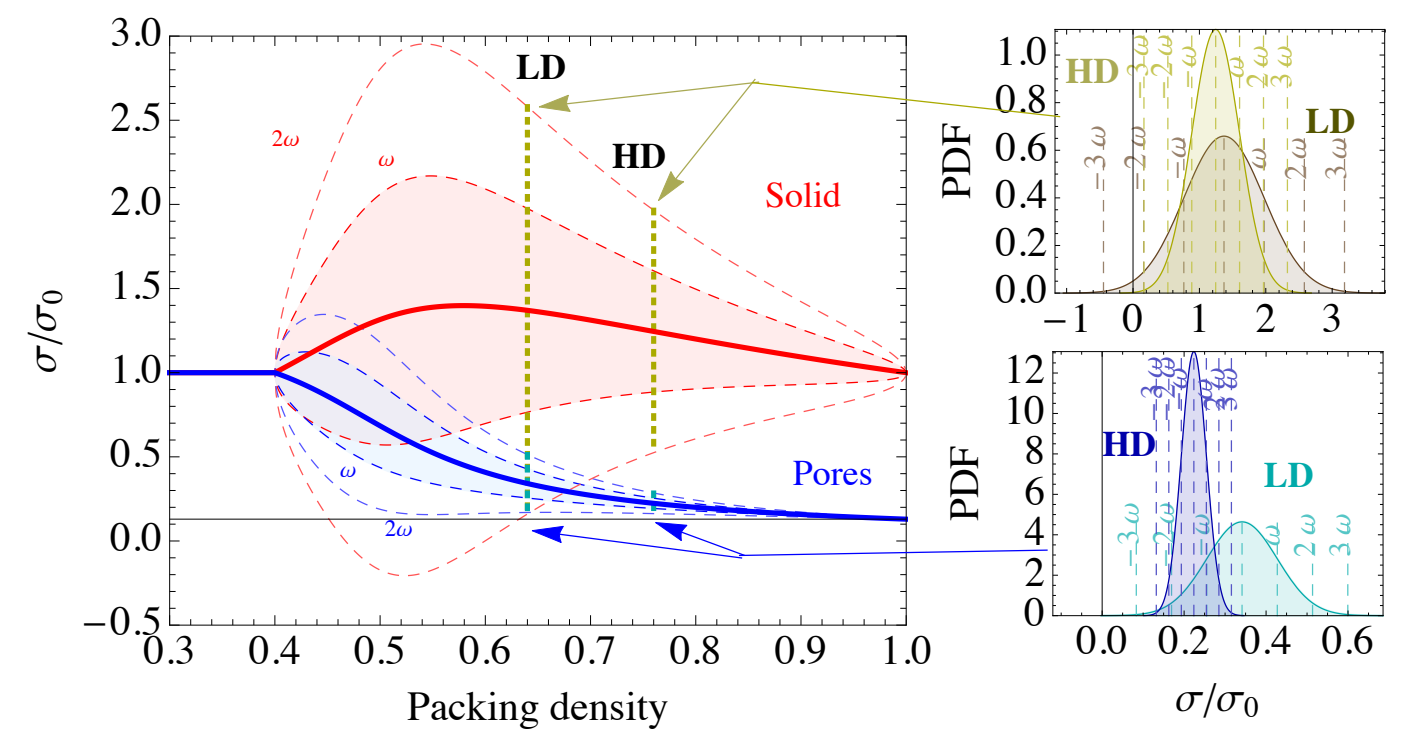

Figure 1. Stress fluctuations within 'undrained' C-S-H according to packing density for a hydrostatic load (left). At right, the PDF at the packing densities corresponding to $\mathrm{HD}$ and LD C-S-H, (0.76 and 0.64, respectively) assuming normal distribution. 


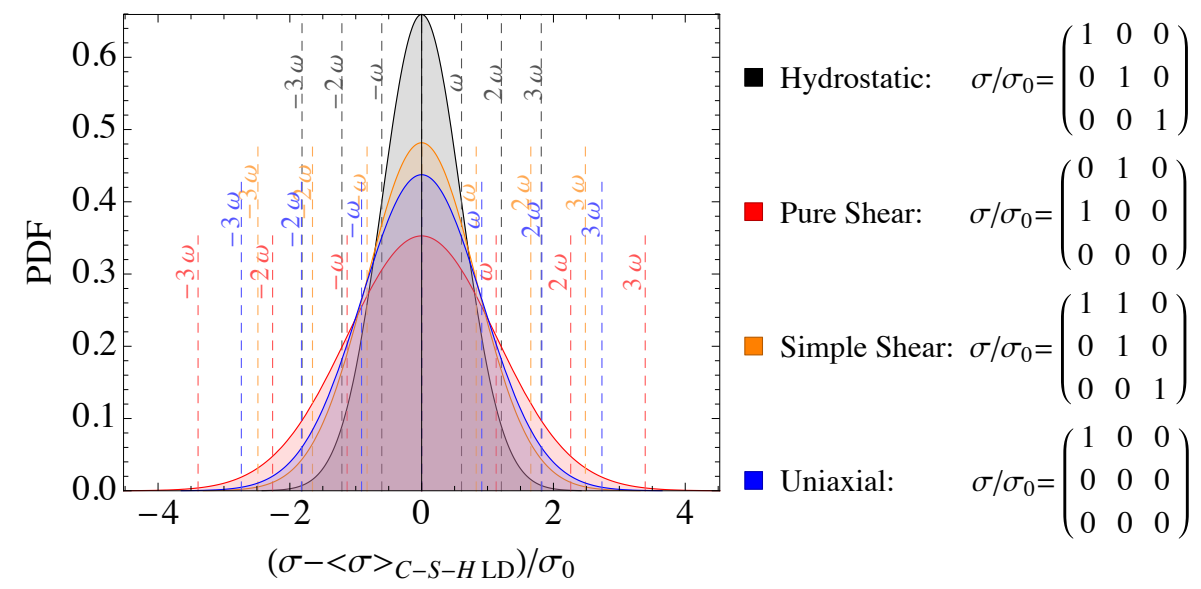

Figure 2. Probability Density Function (PDF - assumed to be Gaussian) of stress fluctuations in LD C-S-H according to loading type.

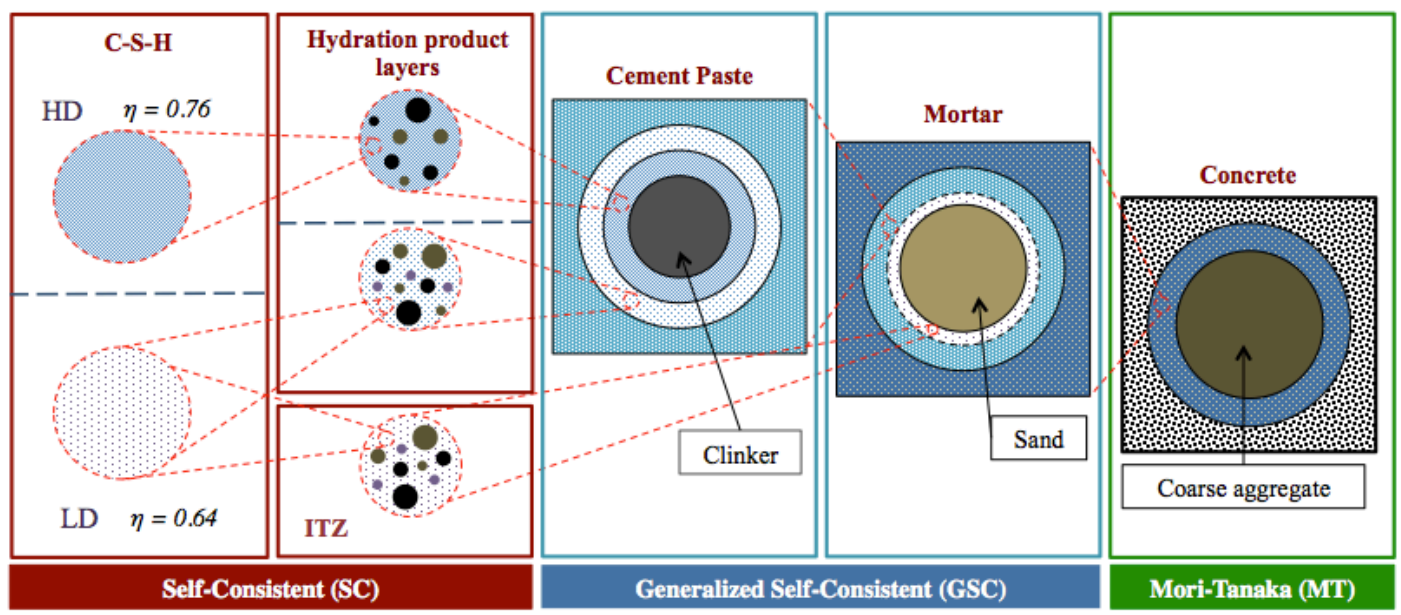

Figure 3. Multiscale representation of the microstructure of CBM.

Estimations of fluctuations in CBM. Keeping the same philosophy of choosing the simplest representation of the microstructure, we provide the estimations of fluctuations for $\mathrm{CBM}$ in a multiscale framework. Figure 3 displays the representation of the hierarchical microstructures of CBM adopted (as in (Honorio et al. under review)). A first stage of homogenization (or upscaling) is necessary to obtain the effective properties in each scale (Figure 4). Then, a second phase of localization (or downscaling) is needed to obtain the average (or interphase) values of the mechanical field of interest. The average values are associated to fluctuations. Thus, in localization processes, fluctuations propagate through smaller scales. In order to account, in a very simplistic way, for the effects of capillary pressure $P_{C}$ due to hydration induced selfdesiccation (which is the transformation field were are considering), we use the expression $\mathrm{P}_{\mathrm{C}}=37.55\left(S^{-\frac{1}{0.46}}-1\right)^{0.54}$ (Coussy et al. 2004), where $S$ is total saturation degree (including gel pores; not discounted chemical shrinkage). Further developments should include different classes of pores with different saturation conditions. 

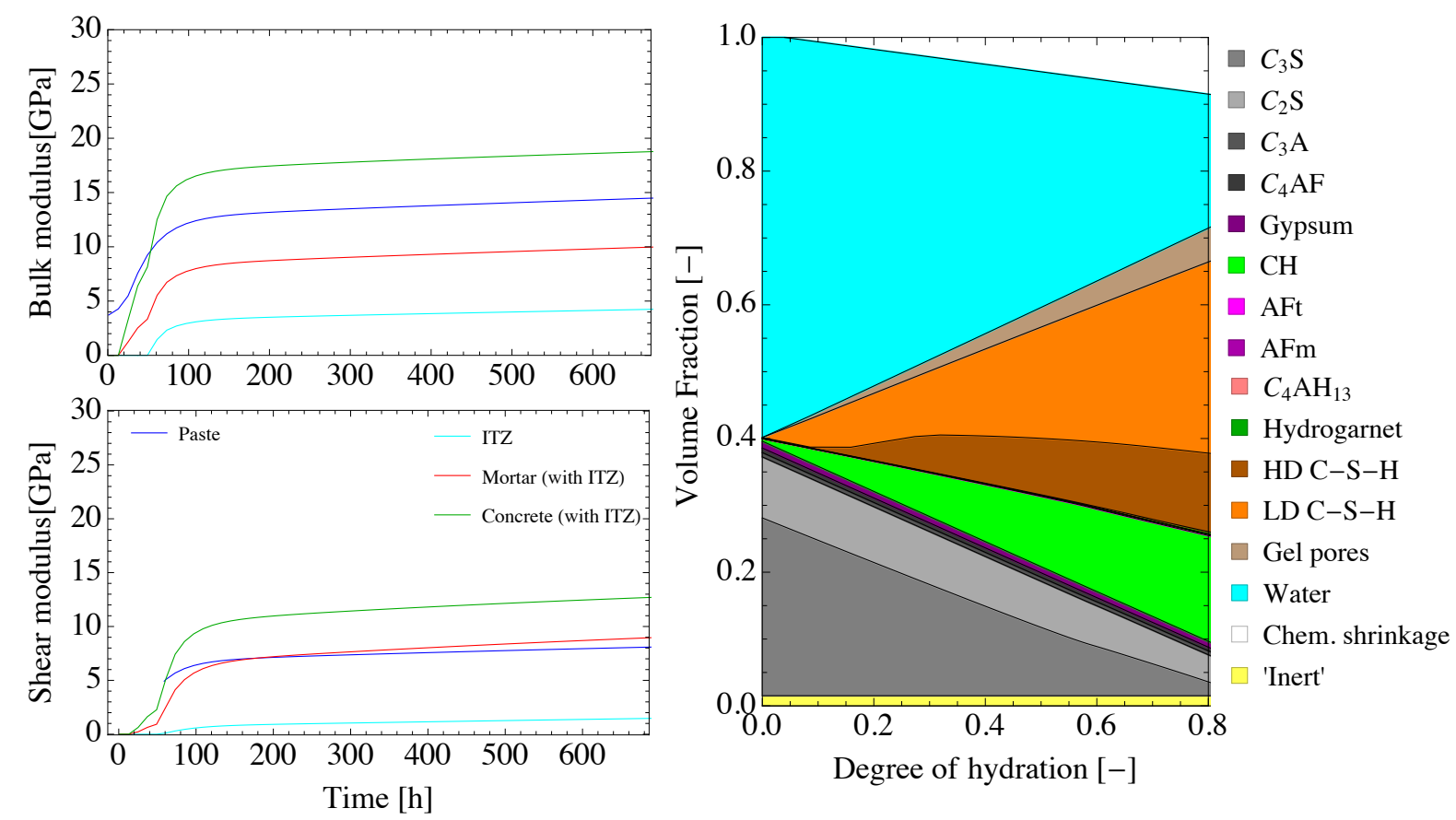

Figure 4. Homogenization of elastic properties at cement paste, ITZ, mortar and concrete scales (left). Evolution of volume fractions of clinker minerals and hydrate products with respect to the degree of hydration (right).

Figure 5 shows the fluctuations (one standard deviation) of stresses in the principal direction for a hydrostatic loading $\sigma_{0}$ in the presence and in the absence of the pressure induced by self-desiccation. The fluctuations, in the presence of pore pressure, are larger than in the absence.

Figure 6 displays the fluctuations (one standard deviation) for the same load at mortar and cement paste scales. Recall that the cement paste, as shown in Figure 3, is represented by two scales. Sand and clinker grains support higher stresses and are subject to higher fluctuations. The leftmost graphic in Figure 6 show that the ITZ is subjected to the lower fluctuations, which are comparable to the ones associated to solid phases at cement paste scale. The rightmost graphic in Figure 6 show the fluctuations for C-S-H HD, Ettringite (AFt), Calcium hydroxide $(\mathrm{CH})$ and water filled pores (the other phases are not shown for clarity). The first three solid phases are subject to similar inter and intra-phase distribution of stresses. As can be seen in the graphic in the center, the fluctuation in HD and LD layers are similar by one $\sigma_{0}$.

The accumulation of fluctuations in downscaling is visible in Figure 5 and 6 . The fluctuations of the clinker grain (the phase with larger fluctuations at cement paste level) are roughly five times the fluctuations of cement paste per se, which is, in turn 3 times the fluctuations of mortar (in the presence of transformation field). 


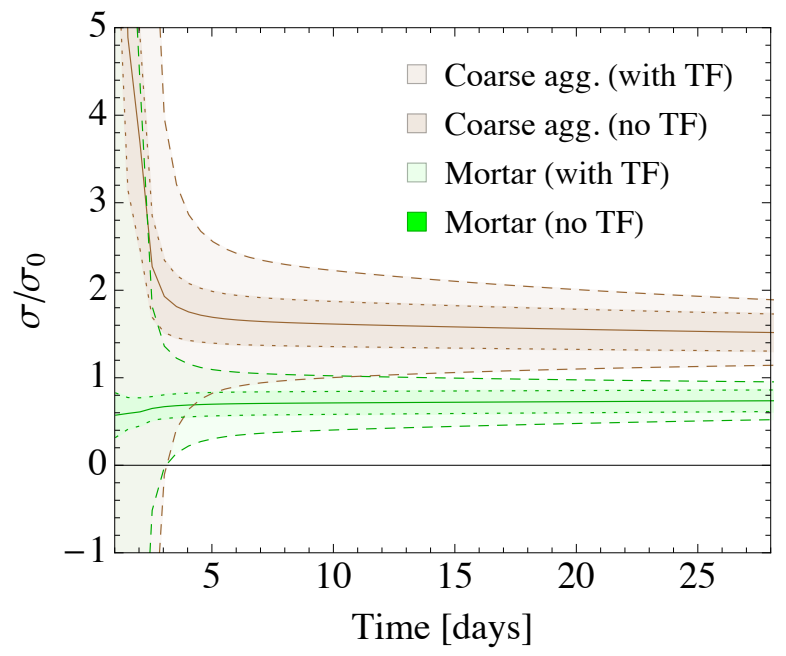

Figure 5. Fluctuations of stresses under hydrostatic loading $\left(\sigma_{0}=1 \mathrm{MPa}\right)$ at concrete scales in the presence and in the absence of the transformation fields (TF).
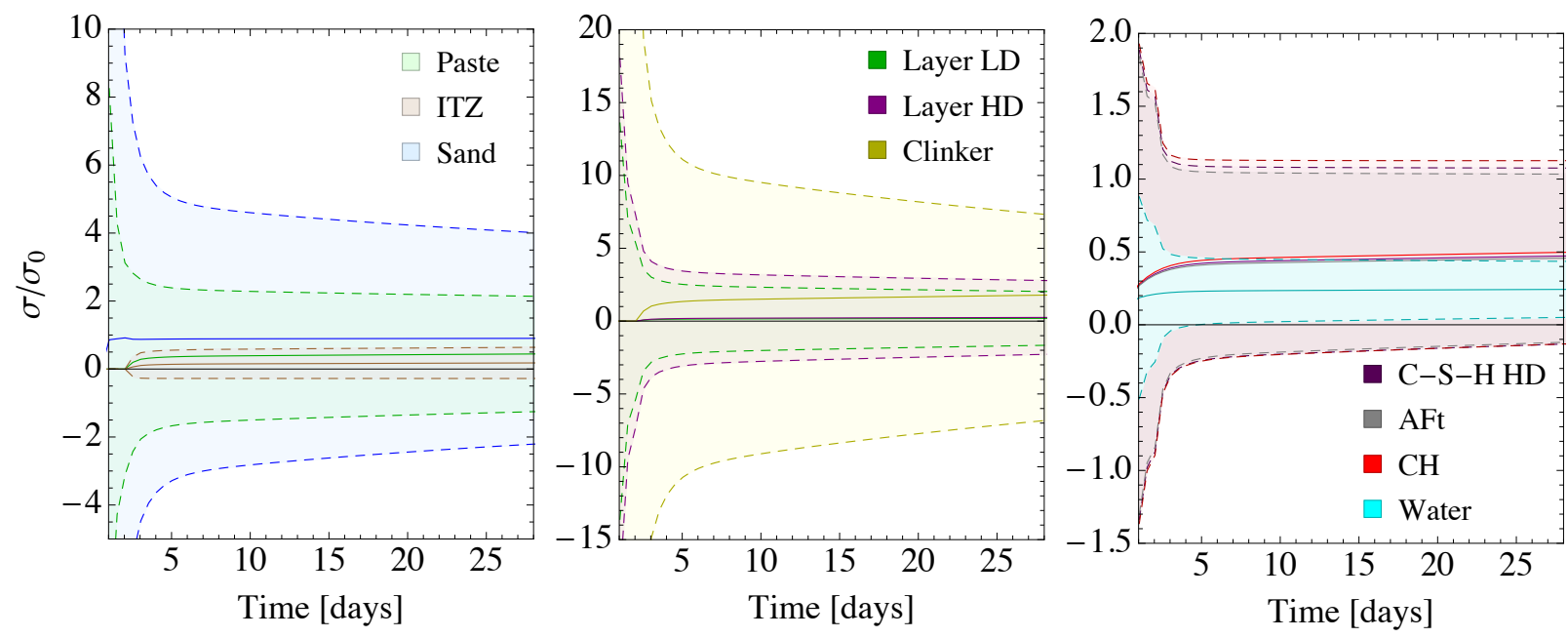

Figure 6. Fluctuations of stresses under hydrostatic loading at mortar and cement paste scales. (Layer HD 1 stands for the layer with HD C-S-H functioning as a matrix; Layer LD, for LD C-S- $\mathrm{H}$ functioning as a matrix)

\section{CONCLUSION}

Estimations of the fluctuations of stress fields in CBM are provided in a multiscale framework. Homogenization schemes in its most basic form (micro/macro isotropic with spherical inclusions) are adopted, so that the estimations do not need to account for the effect of anisotropy or other complexities. Our estimations can be seen as a baseline fluctuation occurring in CBM based mainly on the contrast and interaction of different forms but irrespective of the forms itself. Extensions to estimations in thermo-elasticity are straightforward and treated in a future contribution. 
Acknowledgments. The financial support of the French National Research Agency (ANR) through the project TEAM2ClayDesicc (ANR-14-CE05-0023-01) is gratefully acknowledged.

\section{REFERENCES}

Bobeth, M., and Diener, G. (1986). "Field fluctuations in multicomponent mixtures." Journal of the Mechanics and Physics of Solids, 34(1), 1-17.

Bobeth, M., and Diener, G. (1987). "Static elastic and thermoelastic field fluctuations in multiphase composites." Journal of the Mechanics and Physics of Solids, 35(2), 137-149.

Bornert, M., Masson, R., Castañeda, P. P., and Zaoui, A. (2001). "Second-order estimates for the effective behaviour of viscoplastic polycrystalline materials." Journal of the Mechanics and Physics of Solids, The Jean-Paul Boehler Memorial Volume, 49(11), 2737-2764.

Brenner, R., Castelnau, O., and Badea, L. (2004). "Mechanical field fluctuations in polycrystals estimated by homogenization techniques." Proceedings of the Royal Society of London A: Mathematical, Physical and Engineering Sciences, 460(2052), 3589-3612.

Brenner, R., Castelnau, O., and Gilormini, P. (2001). "A modified affine theory for the overall properties of nonlinear composites." Comptes Rendus de l'Académie des Sciences Series IIB - Mechanics, 329(9), 649-654.

Buryachenko, V. A., and Rammerstorfer, F. G. (1998). "Thermoelastic stress fluctuations in random-structure coated particulate composites." European Journal of Mechanics A/Solids, 17(5), 763-788.

Constantinides, G., and Ulm, F.-J. (2004). "The effect of two types of C-S-H on the elasticity of cement-based materials: Results from nanoindentation and micromechanical modeling." Cement and Concrete Research, 34(1), 67-80.

Coussy, O., Dangla, P., Lassabatère, T., and Baroghel-Bouny, V. (2004). "The equivalent pore pressure and the swelling and shrinkage of cement-based materials." Materials and Structures, 37(1), 15-20.

Dvorak, G. J., and Benveniste, Y. (1992). "On Transformation Strains and Uniform Fields in Multiphase Elastic Media." Proceedings of the Royal Society of London A: Mathematical, Physical and Engineering Sciences, 437(1900), 291-310.

Honorio, T., Bary, B., and Benboudjema, F. (under review). "Thermal properties of cementbased materials: multiscale estimations at early-age."

Honorio, T., Bary, B., and Benboudjema, F. (2016). "Multiscale estimation of the viscoelastic properties of cement-based materials at early age: a combined analytical and numerical approach." Cement and Concrete Research.

Kreher, W., and Pompe, W. (1985). "Field fluctuations in a heterogeneous elastic material—an information theory approach." Journal of the Mechanics and Physics of Solids, 33(5), 419-445.

Laws, N. (1973). "On the thermostatics of composite materials." Journal of the Mechanics and Physics of Solids, 21(1), 9-17.

Ponson, L. (2016). "Statistical aspects in crack growth phenomena: how the fluctuations reveal the failure mechanisms." International Journal of Fracture, 1-17. 